\title{
Minimality of Descriptor Representations under External Equivalence*
}

\author{
M. KUIJPER $\dagger$ and J. M. SCHUMACHER $\ddagger$
}

Considering dynamical systems in terms of their behaviours, the minimality of a descriptor representation is investigated under the corresponding type of equivalence, and a transformation group for minimal descriptor representations is obtained

Key Words-Linear systems; minimal realization; multivariable systems; redundancy; state-space.

\begin{abstract}
Necessary and sufficient conditions are derived for the minimality of descriptor representations under external equivalence. These conditions are stated completely in terms of the matrices $E, A, B, C$ and $D$. Use is made of the close connection between the descriptor representation and the so-called pencil representation. This connection is further exploited to derive the transformation group for minimal descriptor representations. It is shown that the transformations coincide with the operations of strong equivalence as introduced by Verghese et al. (IEEE Trans. Aut. Control, AC-26, 811-831, 1981).
\end{abstract}

\section{INTRODUCTION AND PRELIMINARIES}

IN THIS paper we consider time invariant linear systems represented by

$$
\begin{aligned}
\sigma E \xi & =A \xi+B u \\
y & =C \xi+D u .
\end{aligned}
$$

Here $\sigma$ denotes differentiation or shift, depending on whether one works in continuous or discrete time. The variables $y, u$ and $\xi$ take values in the output space $Y$, the input space $U$ and the descriptor space $X_{d}$, respectively. The codomain of the mappings $E$ and $A$ will be denoted by $X_{e}$ (equation space). The representation (1.1) is called a descriptor representation (D representation). The descriptor form has been found useful for instance in circuit models (Rosenbrock, 1974a), econometric mod-

* Received 9 February 1990; revised 6 August 1990; revised 15 February 1991; received in final form 12 March 1991. The original version of this paper was presented at the IFAC Workshop on System Structure and Control: State Space and Polynomial Methods which was held in Prague, Czechoslovakia during September, 1989. The published proceedings of this IFAC Meeting may be ordered from Pergamon Press plc, Headington Hill Hall, Oxford OX3 $0 \mathrm{BW}$, U.K. This paper was recommended for publication in revised form by Associate Editor V. Kučera.

+CWI, P.O. Box 4079, 1009 AB Amsterdam, The Netherlands. Author to whom all correspondence should be addressed.

$\ddagger C W I$, Amsterdam and Department of Economics, Tilburg University, P.O. Box 90153, 5000 LE Tilburg, The Netherlands. els (Luenberger and Arbel, 1978) and system inversion (Grimm, 1984). The behaviour $\mathscr{B}$ of the system (Willems, 1983, 1986) is, roughly speaking, defined as the set of time trajectories of the input and output variables (the "external variables") that arise from the system representation. We will denote the vector of external variables by $w\left(=\left[y^{\mathrm{T}} u^{\mathrm{T}}\right]^{\mathrm{T}}\right) ; w$ takes its values in $W(=Y \oplus U)$. In order to give a more precise definition of $\mathscr{B}$, we have to make a distinction between the discrete-time case and the continuous-time case. In discrete time $\mathscr{B}$ is defined as a subspace of $W^{\mathbb{Z}_{+}}$:

$$
\begin{array}{r}
\mathscr{B}=\left\{\left(\left(\begin{array}{l}
y_{0} \\
u_{0}
\end{array}\right),\left(\begin{array}{l}
y_{1} \\
u_{1}
\end{array}\right), \ldots\right) \mid \exists\left(\xi_{0}, \xi_{1}, \ldots\right)\right. \\
\text { such that } E \xi_{k+1}=A \xi_{k}+B u_{k} \\
\text { and } \left.y_{k}=C \xi_{k}+D u_{k} \text { for all } k \geqslant 0\right\} .
\end{array}
$$

In continuous time we have to specify function classes to which trajectories should belong. For the sake of simplicity we will work with the class of arbitrarily often differentiable functions on $\mathbb{R}_{+}$. Then the continuous-time behaviour is defined as

$$
\begin{aligned}
& \mathscr{B}=\left\{\left(\left(\begin{array}{l}
y \\
u
\end{array}\right) \in C^{\infty}\left(\mathbb{R}_{+} ; Y \oplus U\right) \mid \exists \xi \in C^{\infty}\left(\mathbb{R}_{+} ; X_{d}\right)\right.\right. \\
& \text { such that } E \dot{\xi}(t)=A \xi(t)+B u(t) \\
& \text { and } \left.y(t)=C \xi(t)+D u(t) \text { for all } t \in \mathbb{R}_{+}\right\} .
\end{aligned}
$$

The notion of behaviour immediately leads to a concept of equivalence.

Definition 1 (Willems, 1983, 1986). Systems are defined to be externally equivalent if their behaviours are the same. 
The aim of this paper is twofold. First, we consider the question under which conditions a D representation of a system is minimal among all other $\mathbf{D}$ representations that are externally equivalent. We will give a characterization of minimality in terms of the matrices $E, A, B, C$ and $D$. Second, we will give the complete set of transformations by which minimal D representations that give rise to the same behaviour can be transformed into each other.

With the latter result we actually provide an operational form of external equivalence for minimal $\mathbf{D}$ representations. In the past, many other concepts of equivalence have been defined for D representations. These were mainly defined in operational form. In this paper the "operations of strong equivalence", as introduced in Verghese et al. (1981), will be important. Verghese et al. defined the concept of "strong equivalence" as a modified version of "restricted system equivalence". Restricted system equivalence was introduced by Rosenbrock (1974b) in an attempt to define a concept of equivalence for $\mathbf{D}$ representations that comes close to "Kalman equivalence" as defined for standard state space representations. However, in his definition the nondynamic variables that can be present in a $\mathbf{D}$ representation were not treated in a satisfactory way. For this reason Verghese et al. defined "strong equivalence". The definition in Verghese et al. (1981) involves the introduction of certain operations on the system matrix of a $\mathbf{D}$ representation which we repeat here.

Definition 2 (Verghese et al., 1981). The modification of the system matrix

to the form

$$
\left[\begin{array}{c|c}
S E-A & -B \\
\hline C & D
\end{array}\right]
$$

$$
\left[\begin{array}{cc|c}
s E-A & 0 & -B \\
0 & I & 0 \\
\hline C & 0 & D
\end{array}\right]
$$

is called a trivial augmentation. The reverse process, corresponding to the deletion of trivial variables, is called a trivial deflation.

Definition 3 (Verghese et al., 1981). The D representations $(E, A, B, C, D)$ and $(\tilde{E}, \tilde{A}, \tilde{B}, \tilde{C}, \tilde{D})$ are related by operations of strong equivalence if there exist matrices $M, N$, $X$ and $Y$ with $M$ and $N$ invertible such that

$$
\begin{aligned}
& {\left[\begin{array}{cc}
M & 0 \\
X & I
\end{array}\right]\left[\begin{array}{cc}
s E-A & -B \\
C & D
\end{array}\right]} \\
& =\left[\begin{array}{cc}
s \tilde{E}-\tilde{A} & -\tilde{B} \\
\tilde{C} & \tilde{D}
\end{array}\right]\left[\begin{array}{cc}
N & Y \\
0 & I
\end{array}\right] .
\end{aligned}
$$

Definition 4 (Verghese et al., 1981). Two D representations are called strongly equivalent if one can be obtained from the other by some sequence of operations of strong equivalence and/or trivial augmentations and/or trivial deflations.

A closed form expression for strong equivalence can also be given. In Pugh et al. (1987) it is proven that the $\mathbf{D}$ representations $(E, A, B$, $C, D)$ and $(\tilde{E}, \tilde{A}, \tilde{B}, \tilde{C}, \tilde{D})$ are strongly equivalent if and only if there exist matrices $M$, $X, \quad X$ and $Y$ such that $[s \tilde{E}-\tilde{A} M]$ and $\left[N^{\mathrm{T}} s E^{\mathrm{T}}-A^{\mathrm{T}}\right]^{\mathrm{T}}$ have neither finite nor infinite zeros and

$$
\begin{aligned}
& {\left[\begin{array}{cc}
M & 0 \\
X & I
\end{array}\right]\left[\begin{array}{cc}
s E-A & -B \\
C & D
\end{array}\right]} \\
& =\left[\begin{array}{cc}
s \tilde{E}-\tilde{A} & -\tilde{B} \\
\tilde{C} & \tilde{D}
\end{array}\right]\left[\begin{array}{cc}
N & Y \\
0 & I
\end{array}\right] .
\end{aligned}
$$

This is called complete equivalence in Pugh et al. (1987). There are several other types of equivalence for $\mathbf{D}$ representations (fundamental equivalence, constant equivalence, etc.) which coincide with strong equivalence. A survey of these can be found in Ferreira (1987).

Apart from external equivalence, there are more types of equivalence that are not defined in operational form. The concept of transfer equivalence arises when the invariant is the transfer function instead of the behaviour. Of course transfer equivalence only applies when the transfer function of the system exists. Grimm (1988) used a generalized notion of transfer equivalence, that is also applicable to systems for which no transfer function exists. In fact, Aplevich $(1981,1985)$ used the same concept (which he named "external equivalence"). In this paper we will call this type of equivalence input-output equivalence.

We will now clarify the relation between external equivalence and the other types of equivalence which are mentioned above. It should be noted that trajectories of the output variables that are not influenced by the input variables (the "uncontrolled behaviour") are invariant under external equivalence: they are, by definition, included in the behaviour of the system. Since such trajectories can be removed under input-output equivalence, it can be shown that external equivalence is stronger than input-output equivalence. On the other hand, nonobservable modes can be removed under external equivalence while they are preserved under strong equivalence. Using this, it can be shown that strong equivalence is stronger than external equivalence.

Our motivation for using external equivalence 
is the following. Behind every notion of system equivalence is the intuitive idea that a system of equations is only a representation of a more intrinsic object. External equivalence defines this "object" as the set of solutions associated with the system. Therefore, this equivalence notion can be applied to any system of equations provided only that a solution concept is defined, which seems the least one can ask. The alternative notion of input-output equivalence is defined by associating a rational vector space to a system of equations; besides looking somewhat less natural and being restricted to linear finite-dimensional systems, this procedure is unable to distinguish, for example, between the systems $\dot{y}=\dot{u}$ and $y=u$.

In order to place the results of this paper into perspective, we will now review the corresponding results for the standard state space case. Note that a standard state-space representation corresponds with a $\mathbf{D}$ representation (1.1) with $E=I$ :

$$
\begin{aligned}
\sigma x & =A x+B u \\
y & =C x+D u .
\end{aligned}
$$

Here $X_{d}=X_{e}=X$ is the state space. The state space representation (1.6) is called minimal under external equivalence if $\operatorname{dim} X$ is minimal among all representations that are externally equivalent. In the following theorem it is stated that observability is a necessary and sufficient condition for minimality under external equivalence.

Theorem 1 (Willems, 1983). A state space representation $(A, B, C, D)$ is minimal under external equivalence if and only if $\left[s I^{\mathrm{T}}-\right.$ $\left.A^{\mathrm{T}} C^{\mathrm{T}}\right]^{\mathrm{T}}$ has full column rank for all $s \in \mathbb{C}$.

It should be noted that controllability is not required for minimality under external equivalence. This is due to the fact that the uncontrolled behaviour remains invariant under external equivalence (see above). Two statespace representations $(A, B, C, D)$ and $(\tilde{A}, \tilde{B}, \tilde{C}, \tilde{D})$ are called isomorphic (or "Kalman equivalent") if there exists an invertible mapping $T$ such that $\tilde{A}=T A T^{-1}, \bar{B}=T B, \tilde{C}=C T^{-1}$ and $\tilde{D}=D$. Theorem 2 can be considered as a state space isomorphism theorem for external equivalence.

Theorem 2 (Willems, 1983). Let $(A, B, C, D)$ be a minimal representation in state space form. A minimal state space representation $(\tilde{A}, \tilde{B}, \tilde{C}, \tilde{D})$ is externally equivalent with $(A, B, C, D)$ if and only if it is isomorphic to $(A, B, C, D)$.
By this theorem, the transformation group for minimal state space representations, i.e. the group of transformations under which minimal state space representations are externally equivalent, coincides with the group of isomorphisms.

We shall now consider the minimality results under input-output equivalence for D representations that exist in the literature. It turns out that different notions of minimality have been defined. Verghese et al. (1981) define minimality in terms of the rank of $E$. They find that a $\mathbf{D}$ representation is minimal under transfer equivalence ( $s E-A$ is assumed to be invertible) if and only if it is strongly irreducible, i.e. reachable and observable at both finite and infinite modes:

- $\left[\begin{array}{lll}s E-A & B\end{array}\right]$ has full row rank for all $s \in \mathbb{C} \cup\{\infty\}$

$-\left[s E^{\mathrm{T}}-A^{\mathrm{T}} C^{\mathrm{T}}\right]^{\mathrm{T}}$ has full column rank for all $s \in \mathbb{C} \cup\{\infty\}$.

It is also shown in (Verghese et al., 1981) that for strongly irreducible representations the transfer function is a complete invariant under strong equivalence. More specifically, it is shown that representations with the same transfer function that are strongly irreducible (and therefore minimal with respect to the rank of $E$ ) are related by operations of strong equivalence. Grimm (1988) takes D representations into account for which $s E-A$ is not necessarily invertible and he defines minimality in terms of the size of $E$. A D representation is then found to be minimal under input-output equivalence if and only if it is strongly irreducible and in addition free of so-called "nondynamic" variables:

$$
A[\operatorname{ker} E] \subset \operatorname{im} E \text {. }
$$

Further, it is shown (Grimm, 1988) that input-output equivalent representations that are minimal with respect to the size of $E$ are related by operations of strong equivalence.

In this paper we will consider D representations without making any assumptions on $s E-A$ being square or invertible. For that reason our definition of minimality is formulated in terms of three indices: the rank of $E$, the column defect of $E$ (dim ker $E$ ) and the row defect of $E$ (codim im $E$ ). We define a D representation to be minimal if each of these three indices is minimal within the set of $\mathbf{D}$ representations that correspond to the same behaviour. In analogy with the standard state space case, one would expect that some kind of observability is required for a $\mathbf{D}$ representation to be minimal under external equivalence. Indeed, observability turns out to be a necessary 
condition. However, more conditions are needed to characterize minimality under external equivalence. In this paper we derive four conditions that are necessary and sufficient. Also, we prove that the transformation group for minimal D representations under external equivalence is again the group of operations of strong equivalence.

In the development below, a prominent role is played by the so-called pencil representation (P representation):

$$
\begin{aligned}
\sigma G z & =F z \\
y & =H_{y} z \\
u & =H_{u} z .
\end{aligned}
$$

Here $F$ and $G$ are linear mappings from $Z$ to $X$, where $Z$ is the space of internal variables and $X$ is the equation space. The pencil form is suited as well for representing systems for which a transfer function does not exist or is nonproper. The representation is called minimal if both $\operatorname{dim} Z$ and $\operatorname{dim} X$ are minimal. It is shown (Willems, 1986; Kuijper and Schumacher, 1990) that a minimal $\mathbf{P}$ representation can be realized directly from the behaviour of the system in a natural way. In the literature, minimality under external equivalence has been characterized in terms of the matrices $F, G, H_{y}$ and $H_{u}$ and the transformation group for minimal $\mathbf{P}$ representations has been derived. In these results the pencil representation is defined by taking the input and output variables together as the vector $w$ of external variables, that was introduced before. A $\mathbf{P}$ representation is then given by

$$
\begin{aligned}
\sigma G z & =F z \\
w & =H z .
\end{aligned}
$$

Before summarizing the existing results, we remark that there are other possibilities of representing a system in general first-order form. Willems (1989) introduced representations of the form

$$
\sigma E x+F x+G w=0 .
$$

A first-order representation of this form has the property that the variable $x$ is a state variable. It should be noted that this is not true for the variable $z$ in our $\mathbf{P}$ representation: the variable $z$ consists of state variables as well as so-called "driving variables" (Willems, 1986).

We now summarize the existing results on $\mathbf{P}$ representations in the following propositions.

Proposition 1 (Kuijper and Schumacher, 1990). A $\mathbf{P}$ representation given by $(F, G, H)$ is minimal under external equivalence if and only if the following conditions hold: (i) $G$ is surjective, (ii) $\left[G^{\mathrm{T}} H^{\mathrm{T}}\right]^{\mathrm{T}}$ is injective, (iii) $\left[s G^{\mathrm{T}}-F^{\mathrm{T}} H^{\mathrm{T}}\right]^{\mathrm{T}}$ has full column rank for all $s \in \mathbb{C}$.

Proposition 2 (Willems, 1983; Kuijper and Schumacher, 1990). Two minimal $\mathbf{P}$ representations $(F, G, H)$ and $(\tilde{F}, \tilde{G}, \tilde{H})$ are externally equivalent if and only if there exist invertible matrices $S$ and $T$ such that

$$
\left[\begin{array}{ll}
S & 0 \\
0 & I
\end{array}\right]\left[\begin{array}{c}
s G-F \\
H
\end{array}\right]=\left[\begin{array}{c}
s \tilde{G}-\tilde{F} \\
\tilde{H}
\end{array}\right] T .
$$

In Kuijper and Schumacher (1990) it is shown that a close connection exists between a $\mathbf{P}$ representation and a $\mathbf{D}$ representation: an algorithm is given for rewriting a $\mathbf{P}$ representation in descriptor form in such a way that minimality is preserved. In the next section we present an algorithm with a similar property for rewriting a $\mathbf{D}$ representation in pencil form. Both algorithms are used for deriving minimality results for $\mathbf{D}$ representations in Section 3. The connection between a $\mathbf{P}$ representation and a $\mathbf{D}$ representation is further exploited in Section 4 to derive the transformation group with respect to external equivalence for minimal D representations.

\section{ALGORITHMS}

In this section we present algorithms for obtaining a D representation from a $\mathbf{P}$ representation and vice versa. These algorithms will be used in the next section where we derive results on the minimality of $\mathbf{D}$ representations by using the known results for $\mathbf{P}$ representations that were mentioned in the introduction. For that reason it is important that both algorithms preserve minimality. There is a trivial way to rewrite a $\mathbf{P}$ representation in descriptor form. Starting from the $\mathbf{P}$ representation $\left(F, G, H_{y}\right.$, $H_{u}$ ), we obtain an equivalent $\mathbf{D}$ representation that is given by

$$
\begin{aligned}
\sigma\left[\begin{array}{l}
G \\
0
\end{array}\right] \xi & =\left[\begin{array}{c}
F \\
H_{u}
\end{array}\right] \xi+\left[\begin{array}{c}
0 \\
-I
\end{array}\right] u \\
y & =H_{y} \xi .
\end{aligned}
$$

However when $\left(F, G, H_{y}, H_{u}\right)$ is minimal, the represention (2.1) is not necessarily minimal. For example, as a starting point one could take a minimal standard state space representation in pencil form, i.e. $G=\left[\begin{array}{ll}I & 0\end{array}\right], \quad F=\left[\begin{array}{ll}A & B\end{array}\right], \quad H_{y}=$ $\left[\begin{array}{ll}C & D\end{array}\right]$ and $H_{u}=\left[\begin{array}{ll}0 & I\end{array}\right]$, which yields the $\mathbf{D}$ representation

$$
\begin{aligned}
\sigma\left[\begin{array}{ll}
I & 0 \\
0 & 0
\end{array}\right]\left(\begin{array}{l}
\xi_{1} \\
\xi_{2}
\end{array}\right) & =\left[\begin{array}{ll}
A & B \\
0 & I
\end{array}\right]\left(\begin{array}{l}
\xi_{1} \\
\xi_{2}
\end{array}\right)+\left[\begin{array}{c}
0 \\
-I
\end{array}\right] u \\
y & =\left[\begin{array}{ll}
C & D
\end{array}\right]\left(\begin{array}{l}
\xi_{1} \\
\xi_{2}
\end{array}\right) .
\end{aligned}
$$


In this case, (2.2) is clearly not a minimal representation. The following algorithm, which already appeared in Kuijper and Schumacher (1990), does a better job at preserving minimality properties.

Algorithm 1. Let a $\mathbf{P}$ representation be given by ( $\left.F, G, H_{y}, H_{u}\right)$. Decompose the internal variable space $Z$ as $Z_{0} \oplus Z_{1} \oplus Z_{2}$ where $Z_{1}=\operatorname{ker} G \cap$ ker $H_{u}$, and $Z_{1} \oplus Z_{2}=\operatorname{ker} G$. Accordingly, write

$$
\begin{aligned}
G & =\left[\begin{array}{lll}
G_{0} & 0 & 0
\end{array}\right], \quad F=\left[\begin{array}{lll}
F_{0} & F_{1} & F_{2}
\end{array}\right], \\
H_{y} & =\left[\begin{array}{llll}
H_{00} & H_{01} & H_{02}
\end{array}\right], \quad H_{u}=\left[\begin{array}{llll}
H_{u 0} & 0 & H_{u 2}
\end{array}\right] .
\end{aligned}
$$

The matrix $H_{u 2}$ has full column rank, and by renumbering the $u$ variables if necessary, we can write

$$
H_{u 0}=\left[\begin{array}{l}
H_{10} \\
H_{20}
\end{array}\right], \quad H_{u 2}=\left[\begin{array}{l}
H_{12} \\
H_{22}
\end{array}\right]
$$

where $H_{22}$ is invertible (or empty, if $\operatorname{ker} G \subset$ ker $H_{u}$ ). Define descriptor matrices by

$$
\begin{gathered}
E=\left[\begin{array}{cc}
G_{0} & 0 \\
0 & 0
\end{array}\right], \quad A=\left[\begin{array}{cc}
\bar{F}_{0} & F_{1} \\
\bar{H}_{10} & 0
\end{array}\right], \\
B=\left[\begin{array}{cc}
0 & \bar{F}_{2} \\
-I & \bar{H}_{12}
\end{array}\right], \quad C=\left[\begin{array}{ll}
\bar{H}_{00} & H_{01}
\end{array}\right], \\
D=\left[\begin{array}{ll}
0 & \bar{H}_{02}
\end{array}\right]
\end{gathered}
$$

with

$$
\begin{aligned}
\bar{F}_{0} & =F_{0}-F_{2} H_{22}^{-1} H_{20} \\
\bar{H}_{00} & =H_{00}-H_{02} H_{22}^{-1} H_{20} \\
\bar{H}_{10} & =H_{10}-H_{12} H_{22}^{-1} H_{20} \\
\bar{F}_{2} & =F_{2} H_{22}^{-1} \\
\bar{H}_{02} & =H_{02} H_{22}^{-1} \\
\bar{H}_{12} & =H_{12} H_{22}^{-1} .
\end{aligned}
$$

The essence of the above construction is that as many $z$ variables as possible are replaced by $u$ variables, while at the same time a minimum number of $y$ variables is introduced as "descriptor state variables". In the following lemma we make precise how certain properties are transformed under the algorithm. Later it will become clear that these properties are actually minimality properties.

Lemma 1. Let $(E, A, B, C, D)$ be a $\mathbf{D}$ representation that results from applying Algorithm 1 to a $\mathbf{P}$ representation, given by $(F, G$, $\left.H_{y}, H_{u}\right)$. Then the two representations are externally equivalent, and furthermore the following holds:

(i) $\operatorname{rank} E=\operatorname{rank} G$

(ii) $\operatorname{dim} \operatorname{ker} E=\operatorname{dim}(Y \cap H[\operatorname{ker} G])+$ $\operatorname{dim} \operatorname{ker}\left[\begin{array}{ll}G^{\mathrm{T}} & H^{\mathrm{T}}\end{array}\right]^{\mathrm{T}}$ (iii) $\operatorname{codim} \operatorname{im} E=\operatorname{codim}(Y+H[\operatorname{ker} G])+$ codim im $G$

(iv) $\operatorname{dim} \operatorname{ker}\left[\begin{array}{ll}E^{\mathrm{T}} & C^{\mathrm{T}}\end{array}\right]^{\mathrm{T}}=\operatorname{dim} \operatorname{ker}\left[\begin{array}{ll}G^{\mathrm{T}} & H^{\mathrm{T}}\end{array}\right]^{\mathrm{T}}$

(v) $\operatorname{codim} \operatorname{im}\left[\begin{array}{ll}E & B\end{array}\right] \leqslant \operatorname{codim} \operatorname{im} G$

Moreover we have the following implications:

(vi) $G$ is surjective $\Rightarrow[E B]$ is surjective

(vii) $\left[G^{\mathrm{T}} H^{\mathrm{T}}\right]^{\mathrm{T}}$ is injective $\Rightarrow\left[E^{\mathrm{T}} C^{\mathrm{T}}\right]^{\mathrm{T}}$ is injective

(viii) $\left[s G^{\mathrm{T}}-F^{\mathrm{T}} H^{\mathrm{T}}\right]^{\mathrm{T}}$ has full column rank for all $s \in \mathbb{C} \Rightarrow\left[s E^{\mathrm{T}}-A^{\mathrm{T}} C^{\mathrm{T}}\right]^{\mathrm{T}}$ has full column rank for all $s \in \mathbb{C}$.

Proof. The only operations that are involved in Algorithm 1 are: choosing another basis for the internal variables; reordering $u$ components; and multiplying an equation from the left by a constant invertible matrix. It is therefore immediate (Schumacher, 1988) that the resulting D representation is externally equivalent with $\left(F, G, H_{y}, H_{u}\right)$. Further, equality (i) is trivial while (ii) follows from

$$
\begin{aligned}
\operatorname{dim} \operatorname{ker} E= & \operatorname{dim}\left(\operatorname{ker} G \cap \operatorname{ker} H_{u}\right) \\
= & \operatorname{dim}(\operatorname{ker} G \cap \operatorname{ker} H) \\
& +\operatorname{dim}(Y \cap H[\operatorname{ker} G]) \\
= & \operatorname{dim} \operatorname{ker}\left[G^{\mathrm{T}} H^{\mathrm{T}}\right]^{\mathrm{T}} \\
& +\operatorname{dim}(Y \cap H[\operatorname{ker} G]) .
\end{aligned}
$$

Denoting the number of rows of $H_{12}$ by $m_{1}$ we have

$$
\begin{aligned}
\operatorname{codim} \operatorname{im} E= & \operatorname{codimim} G_{0}+m_{1} \\
= & \operatorname{codimim} G \\
& +\operatorname{codim}(Y+H[\operatorname{ker} G]) .
\end{aligned}
$$

This implies (iii). Equality (iv) is again trivial while (v) follows from

$$
\begin{aligned}
\operatorname{codim} \operatorname{im}\left[\begin{array}{ll}
E & B
\end{array}\right] & =\operatorname{codim} \operatorname{im}\left[\begin{array}{cccc}
G_{0} & 0 & 0 & \bar{F}_{2} \\
0 & 0 & -I & \bar{H}_{12}
\end{array}\right] \\
& =\operatorname{codimim}\left[\begin{array}{ccc}
G & F_{2} H_{22}^{-1}
\end{array}\right] \\
& \leqslant \operatorname{codimim} G .
\end{aligned}
$$

The implications (vi) and (vii) follow immediately from (v) and (iv), respectively. Implication (viii) can be easily verified by considering the matrix equality

$$
\begin{aligned}
& {\left[\begin{array}{cc}
s G_{0}-\bar{F}_{0} & -F_{1} \\
-\bar{H}_{10} & 0 \\
\bar{H}_{00} & H_{01} \\
0 & 0
\end{array}\right]=\left[\begin{array}{cccc}
I & 0 & 0 & 0 \\
0 & 0 & -I & 0 \\
0 & I & 0 & 0 \\
0 & 0 & 0 & I
\end{array}\right]} \\
& \quad \times\left[\begin{array}{ccc}
s G-F_{0} & -F_{1} & -F_{2} \\
H_{00} & H_{01} & H_{02} \\
H_{10} & 0 & H_{12} \\
H_{20} & 0 & H_{22}
\end{array}\right]\left[\begin{array}{cc}
I & 0 \\
0 & I \\
-H_{22}^{-1} H_{20} & 0
\end{array}\right] .
\end{aligned}
$$


Next we present an algorithm for obtaining a $\mathbf{P}$ representation from a $\mathbf{D}$ representation.

Algorithm 2. Let a $\mathbf{D}$ representation be given by ( $E, A, B, C, D)$. Decompose the descriptor space $X_{d}$ as $X_{d 1} \oplus X_{d 2}$ where $X_{d 2}=\operatorname{ker} E$. Decompose the equation space $X_{e}$ as $X_{e 1} \oplus$ $X_{e 2} \oplus X_{e 3}$ where $X_{e 1}=\operatorname{im} E$ and $X_{e 1} \oplus X_{e 2}=$ $\operatorname{im}\left[\begin{array}{ll}E & B\end{array}\right]$. Accordingly write

$$
\begin{gathered}
E=\left[\begin{array}{ll}
I & 0 \\
0 & 0 \\
0 & 0
\end{array}\right], \quad A=\left[\begin{array}{ll}
A_{11} & A_{12} \\
A_{21} & A_{22} \\
A_{31} & A_{32}
\end{array}\right], \\
B=\left[\begin{array}{c}
B_{1} \\
B_{2} \\
0
\end{array}\right], \quad C=\left[\begin{array}{ll}
C_{1} & C_{2}
\end{array}\right] .
\end{gathered}
$$

The matrix $B_{2}$ is now surjective. By renumbering the $u$ variables if necessary, we can write

$$
\left[\begin{array}{c}
B_{1} \\
B_{2} \\
0
\end{array}\right]=\left[\begin{array}{cc}
B_{11} & B_{12} \\
B_{21} & B_{22} \\
0 & 0
\end{array}\right], \quad D=\left[\begin{array}{ll}
D_{1} & D_{2}
\end{array}\right]
$$

where $B_{22}$ is invertible. Define pencil matrices as:

$$
\begin{gathered}
G=\left[\begin{array}{lll}
I & 0 & 0 \\
0 & 0 & 0
\end{array}\right], \quad F=\left[\begin{array}{ccc}
\bar{A}_{11} & \bar{A}_{12} & \bar{B}_{11} \\
A_{31} & A_{32} & 0
\end{array}\right], \\
H_{y}=\left[\begin{array}{lll}
\bar{C}_{1} & \bar{C}_{2} & \bar{D}_{1}
\end{array}\right], \quad H_{u}=\left[\begin{array}{ccc}
0 & 0 & I \\
\bar{A}_{21} & \bar{A}_{22} & \bar{B}_{21}
\end{array}\right]
\end{gathered}
$$

with

$$
\begin{aligned}
& \bar{A}_{11}=A_{11}-B_{12} B_{22}^{-1} A_{21} \\
& \bar{A}_{12}=A_{12}-B_{12} B_{22}^{-1} A_{22} \\
& \bar{B}_{11}=B_{11}-B_{12} B_{22}^{-1} B_{21} \\
& \bar{C}_{1}=C_{1}-D_{2} B_{22}^{-1} A_{21} \\
& \bar{C}_{2}=C_{2}-D_{2} B_{22}^{-1} A_{22} \\
& \bar{D}_{1}=D_{1}-D_{2} B_{22}^{-1} B_{21} \\
& \bar{A}_{21}=-B_{22}^{-1} A_{21} \\
& \bar{A}_{22}=-B_{22}^{-1} A_{22} \\
& \bar{B}_{21}=-B_{22}^{-1} B_{21} .
\end{aligned}
$$

Lemma 2. Let $\left(F, G, H_{y}, H_{u}\right)$ be a $\mathbf{P}$ representation that results from applying Algorithm 2 to a $\mathbf{D}$ representation, given by ( $E, A, B, C, D)$. Then the two representations are externally equivalent, and furthermore the following equalities hold:

(i) $\operatorname{rank} G=\operatorname{rank} E$

(ii) codim im $G=\operatorname{codim} \operatorname{im}\left[\begin{array}{ll}E & B\end{array}\right]$

(iii) $\operatorname{dim} \operatorname{ker} G=\operatorname{dim} \operatorname{ker} E+\operatorname{dim} B^{-1}[\operatorname{im} E]$

(iv) $\operatorname{dim} \operatorname{ker}\left[G^{\mathrm{T}} H^{\mathrm{T}}\right]^{\mathrm{T}}=\operatorname{dim}(\operatorname{ker} E \cap \operatorname{ker} C \cap$ $\left.A^{-1}\left[\operatorname{im} E \oplus X_{e 3}\right]\right)$
Moreover we have the following implications:

(v) $\left[\begin{array}{ll}E & B\end{array}\right]$ is surjective $\Rightarrow G$ is surjective

(vi) $\operatorname{ker} E \cap \operatorname{ker} C \cap A^{-1}\left[\operatorname{im} E \oplus X_{e 3}\right]=\{0\} \Rightarrow$ $\left[G^{\mathrm{T}} H^{\mathrm{T}}\right]^{\mathrm{T}}$ is injective

(vii) $\left[s E^{\mathrm{T}}-A^{\mathrm{T}} C^{\mathrm{T}}\right]^{\mathrm{T}}$ has full column rank for all $s \in \mathbb{C} \Rightarrow\left[s G^{\mathrm{T}}-F^{\mathrm{T}} H^{\mathrm{T}}\right]^{\mathrm{T}}$ has full column rank for all $s \in \mathbb{C}$.

Proof. The external equivalence of the two representations follows from the same argument as in the proof of the previous lemma. Next, the equalities (i) and (ii) are immediate. Denoting the number of columns of $B_{21}$ by $m_{1}$, equality (iii) follows from:

$$
\begin{aligned}
\operatorname{dim} \operatorname{ker} G= & \operatorname{dim} \operatorname{ker} E+m_{1} \\
= & \operatorname{dim} \operatorname{ker} E+\operatorname{dim} U \\
& -\operatorname{dim} \operatorname{im}\left[\begin{array}{ll}
B_{21} & B_{22}
\end{array}\right] \\
= & \operatorname{dim} \operatorname{ker} E+\operatorname{dim} \operatorname{ker}\left[\begin{array}{ll}
B_{21} & B_{22}
\end{array}\right] \\
= & \operatorname{dim} \operatorname{ker} E+\operatorname{dim} B^{-1}[\operatorname{im} E] .
\end{aligned}
$$

Equality (iv) follows from:

$$
\begin{aligned}
\operatorname{dim} \operatorname{ker}\left[\begin{array}{c}
G \\
H
\end{array}\right]= & \operatorname{dim} \operatorname{ker}\left[\begin{array}{l}
\bar{C}_{2} \\
\bar{A}_{22}
\end{array}\right] \\
= & \operatorname{dim} \operatorname{ker}\left[\begin{array}{cc}
I & -D_{2} B_{22}^{-1} \\
0 & -B_{22}^{-1}
\end{array}\right]\left[\begin{array}{c}
C_{2} \\
A_{22}
\end{array}\right] \\
= & \operatorname{dim} \operatorname{ker}\left[\begin{array}{c}
C_{2} \\
A_{22}
\end{array}\right] \\
= & \operatorname{dim}(\operatorname{ker} E \cap \operatorname{ker} C \\
& \left.\cap A^{-1}\left[\operatorname{im} E \oplus X_{e 3}\right]\right) .
\end{aligned}
$$

The implications (v) and (vi) follow immediately from (ii) and (iv), respectively. Implication (vii) can be easily verified by considering the matrix equality

$$
\begin{aligned}
& {\left[\begin{array}{ccc}
s I-\bar{A}_{11} & -\bar{A}_{12} & -\bar{B}_{11} \\
-A_{31} & -A_{32} & 0 \\
\bar{C}_{1} & \bar{C}_{2} & \bar{D}_{1} \\
0 & 0 & I \\
\bar{A}_{21} & \bar{A}_{22} & \bar{B}_{21}
\end{array}\right] } \\
& {\left[\begin{array}{ccccc}
I & -B_{12} B_{22}^{-1} & 0 & 0 & 0 \\
0 & 0 & I & 0 & 0 \\
0 & D_{2} B_{22}^{-1} & 0 & I & 0 \\
0 & 0 & 0 & 0 & I \\
0 & B_{22}^{-1} & 0 & 0 & 0
\end{array}\right] } \\
& \times\left[\begin{array}{cccc}
s I-A_{11} & -A_{12} & -B_{11} \\
-A_{21} & -A_{22} & -B_{21} \\
-A_{31} & -A_{32} & 0 \\
C_{1} & C_{2} & D_{1} \\
0 & 0 & I
\end{array}\right] .
\end{aligned}
$$




\section{MINIMALITY}

In this section, we derive necessary and sufficient conditions for the minimality under external equivalence of a $\mathbf{D}$ representation $(E, A, B, C, D)$ that are stated completely in terms of the matrices $E, A, B, C$ and $D$. In Kuijper and Schumacher (1990), we considered minimality in a polynomial setting. There we assumed that the system is also represented by a set of autoregressive equations:

$$
R_{1}(\sigma) y+R_{2}(\sigma) u=0
$$

where $R_{1}(s)$ and $R_{2}(s)$ are polynomial matrices. Denoting the space of proper rational $W$ valued functions by $W \llbracket \lambda^{-1} \rrbracket$ and an element of $W \llbracket \lambda^{-1} \rrbracket$ by $w(\lambda)$, we defined the following subspace $W^{0} \subset W$ [see also Willems (1986)]:

$$
\begin{gathered}
W^{0}=\left\{w \in W \mid \exists w(\lambda) \in W \llbracket \lambda^{-1} \rrbracket\right. \text { such that } \\
w(\lambda) \in \operatorname{ker} R(\lambda) \text { and } w=w(\infty)\} .
\end{gathered}
$$

In the following theorem $n$ denotes the sum of the minimal row indices of the matrix $\left[R_{1}(s) R_{2}(s)\right]$.

Theorem 3 (Kuijper and Schumacher, 1990). Let a $\mathbf{D}$ representation be given by $(E, A, B, C$, $D)$. The representation is minimal under external equivalence if and only if

(i) $\operatorname{rank} E=n$

(ii) $\operatorname{dim} \operatorname{ker} E=\operatorname{dim}\left(Y \cap W^{0}\right)$

(iii) $\operatorname{codim}$ im $E=\operatorname{codim}\left(Y+W^{0}\right)$.

Intuitively speaking, the subspace $W^{0}$ is spanned by the minimum number of "driving variables" of the system; when $W^{0}$ coincides with the input space we are dealing with a system with a strictly causal input-output structure. As already mentioned in the introduction, a $\mathbf{P}$ representation $(F, G, H)$ can be obtained directly from the behaviour. It is therefore not surprising that $W^{0}$ can be easily expressed in terms of the matrices $F, G$ and $H$. In Kuijper and Schumacher (1990) we proved the following proposition.

Proposition 3 (Kuijper and Schumacher, 1990). Assume that a $\mathbf{P}$ representation, given by $(F, G, H)$, satisfies the following conditions:

(i) $G$ is surjecture

(ii) $\left[G^{\mathrm{T}} H^{\mathrm{T}}\right]^{\mathrm{T}}$ is injective.

Then we have

$$
W^{0}=H[\operatorname{ker} G] .
$$

Using the above proposition together with the properties of Algorithm 2 as expressed in Lemma 2 we are now able to express $W^{0}$ in terms of the matrices of a $\mathbf{D}$ representation. In the following lemma the mapping $\pi_{U}: W \rightarrow U$ denotes projection onto $U$ along $Y$.
Lemma 3. Assume that a $\mathbf{D}$ representation, given by $(E, A, B, C, D)$, satisfies the following conditions:

(i) $\left[\begin{array}{ll}E & B\end{array}\right]$ is surjective

(ii) $\left[E^{\mathrm{T}} C^{\mathrm{T}}\right]^{\mathrm{T}}$ is injective

(iii) $A[\operatorname{ker} E] \subset \operatorname{im} E$.

Then we have

$$
\begin{aligned}
& Y \cap W^{0}=C[\operatorname{ker} E] \\
& \pi_{U} W^{0}=B^{-1}[\operatorname{im} E] .
\end{aligned}
$$

Proof. Application of Algorithm 2 to our D representation yields a $\mathbf{P}$ representation that, according to Lemma 2, satisfies the conditions of Proposition 3. We may therefore conclude that

$$
W^{0}=\operatorname{im}\left[\begin{array}{cc}
C_{2} & D_{1}-D_{2} B_{22}^{-1} B_{21} \\
0 & I \\
0 & -B_{22}^{-1} B_{21}
\end{array}\right],
$$

where the matrices are partitioned as in Algorithm 2. The equations (3.3) and (3.4) now follow immediately.

In Kuijper and Schumacher (1990) we showed that conditions (i) and (ii) of the above lemma are necessary conditions for the minimality of a D representation. We now prove that this also holds true for condition (iii).

Lemma 4. If the $\mathbf{D}$ representation, given by $(E, A, B, C, D)$, is minimal under external equivalence, then the following holds:

$$
A[\operatorname{ker} E] \subset \operatorname{im} E \text {. }
$$

Proof. Suppose that the condition is not fulfilled, while $(E, A, B, C, D)$ is minimal. Then, by a suitable choice of coordinates, we can represent the system as:

$$
\begin{gathered}
\sigma \xi_{1}=A_{11} \xi_{1}+A_{12} \xi_{2}+B_{1} u \\
0=A_{21} \xi_{1}+A_{22} \xi_{2}+B_{2} u \\
y=C_{1} \xi_{1}+C_{2} \xi_{2}+D u .
\end{gathered}
$$

We now have $A_{22} \neq 0$ and without restrictions we can assume that $A_{22}$ is of the form $\left[\begin{array}{ll}I & 0 \\ 0 & 0\end{array}\right]$. Equation (3.8) then splits into two equations:

$$
\begin{gathered}
0=A_{211} \xi_{1}+\xi_{2}+B_{21} u \\
0=A_{212} \xi_{1}+B_{22} u .
\end{gathered}
$$

Expressing $\xi_{2}$ in $\xi_{1}$ and $u$ and substituting this expression into equations (3.7) and (3.9) leads to an equivalent $\mathbf{D}$ representation $(\tilde{E}, \tilde{A}, \tilde{B}, \tilde{C}, \tilde{D})$ for which

$$
\operatorname{dim} \operatorname{ker} \tilde{E}=\operatorname{dim} \operatorname{ker} E-\operatorname{rank} A_{22}
$$

$\operatorname{codim} \operatorname{im} \tilde{E}=\operatorname{codim} \operatorname{im} E-\operatorname{rank} A_{22}$. 
Since we supposed that rank $A_{22}>0$, this contradicts our assumption on the minimality of $(E, A, B, C, D)$.

Remark 1. In Verghese et al., a D representation $(E, A, B, C, D)$ is defined to contain nondynamic modes if there exist invertible constant matrices $M$ and $N$ such that

$$
M[s E-A] N=\left[\begin{array}{cc}
s \tilde{E}-\tilde{A} & 0 \\
0 & I
\end{array}\right]
$$

where $I$ is an identity matrix of appropriate size. It is not difficult to show that a $\mathbf{D}$ representation contains nondynamic modes if and only if it does not satisfy the condition of the above lemma. Grimm (1988) also made a remark on this.

Remark 2. In the literature (see Lewis, 1986, and references therein) various definitions of observability and controllability/reachability at infinity for $\mathbf{D}$ representations were given. It is easy to see that these definitions coincide when there are no nondynamic modes.

In order to derive the main theorem of this section we will use the characterization of minimality for $\mathbf{P}$ representations that was mentioned in the introduction. As stated before, the algorithms of Section 2 will be used for that purpose and it is therefore important that they preserve minimality. In Kuijper and Schumacher (1990) we already showed that this is the case for Algorithm 1. We now prove that the same property holds for Algorithm 2.

Lemma 5. Let $(F, G, H)$ be a $\mathbf{P}$ representation that results from applying Algorithm 2 to a $\mathbf{D}$ representation that is minimal under external equivalence. Then $(F, G, H)$ is also minimal under external equivalence.

Proof. From Lemma 2 it follows that $G$ is surjective. Furthermore the minimality of rank $G$ follows immediately from the minimality of rank $E$ since in both Algorithm 1 and Algorithm 2 we have

$$
\text { rank } G=\operatorname{rank} E .
$$

In order to conclude that the $\mathbf{P}$ representation is minimal we still have to prove that $\operatorname{dim} \operatorname{ker} G$ is minimal. Using Lemma 3 together with Lemma 2 , we have

$$
\begin{aligned}
\operatorname{dim} \operatorname{ker} G & =\operatorname{dim} \operatorname{ker} E+\operatorname{dim} B^{-1}[\operatorname{im} E] \\
& =\operatorname{dim} C[\operatorname{ker} E]+\operatorname{dim} B^{-1}[\operatorname{im} E] \\
& =\operatorname{dim}\left(Y \cap W^{0}\right)+\operatorname{dim}\left(\pi_{U} W^{0}\right) \\
& =\operatorname{dim} W^{0} .
\end{aligned}
$$

From Kuijper and Schumacher (1990) we may now conclude that $\operatorname{dim} \operatorname{ker} G$ is minimal and moreover that the $\mathbf{P}$ representation $(F, G, H)$ is minimal.

We now present the first main result of this paper.

Theorem 4 . Let a $\mathbf{D}$ representation be given by ( $E, A, B, C, D)$. The representation is minimal under external equivalence if and only if the following conditions hold:

(i) $\left[\begin{array}{ll}E & B\end{array}\right]$ is surjective

(ii) $\left[E^{\mathrm{T}} C^{\mathrm{T}}\right]^{\mathrm{T}}$ is injective

(iii) $A[\operatorname{ker} E] \subset \operatorname{im} E$

(iv) $\left[s E^{\mathrm{T}}-A^{\mathrm{T}} C^{\mathrm{T}}\right]^{\mathrm{T}}$ has full column rank for all $s \in \mathbb{C}$.

Proof. From Lemma 4 and the remark preceding it, it follows immediately that for a minimal D representation the conditions (i), (ii) and (iii) should hold. In order to prove (iv) we apply Algorithm 2 to the representation. According to Lemma 5 the $\mathbf{P}$ representation $(F, G, H)$ that is obtained in this way is minimal. This implies that $\left[s G^{\mathrm{T}}-F^{\mathrm{T}} H^{\mathrm{T}}\right]^{\mathrm{T}}$ should have full column rank for all $s \in \mathbb{C}$ (Proposition 1). Condition (iv) now easily follows from matrix equality (2.14). Conversely, when Algorithm 2 is applied to a D representation for which conditions (i)-(iv) hold, Lemma 2 yields that the resulting $\mathbf{P}$ representation satisfies the conditions of Proposition 1 and is therefore minimal. From this it follows that rank $E$ is minimal. Furthermore since conditions (i), (ii) and (iii) are assumed to be satisfied we can use Lemma 3 to derive

and

$$
\begin{aligned}
\operatorname{dim} \operatorname{ker} E & =\operatorname{dim} C[\operatorname{ker} E] \\
& =\operatorname{dim} Y \cap W^{0}
\end{aligned}
$$

$$
\begin{aligned}
\operatorname{codim} \operatorname{im} E & =\operatorname{codim} B^{-1}[\operatorname{im} E] \\
& =\operatorname{codim} \pi_{U} W^{0} .
\end{aligned}
$$

By Theorem 3 this proves that the D representation is minimal.

\section{TRANSFORMATIONS}

Grimm (1988) proves that minimal D representations, that are input-output equivalent, are related by operations of strong equivalence. In this section we present a similar result in our context of external equivalence. We first prove that strong equivalence implies external equivalence. As already mentioned in the introduction, it can in fact be shown that strong equivalence is a strictly stronger concept than external equivalence since nonobservable finite modes are invariant under strong equivalence while they can be eliminated under external equivalence. 
Proposition 4. Let $(E, A, B, C, D)$ and $(\tilde{E}, \tilde{A}, \tilde{B}, \tilde{C}, \tilde{D})$ be $\mathbf{D}$ representations that are strongly equivalent. Then the two representations are externally equivalent.

Proof. It is clear that "trivial augmentations" and "trivial deflations" do not affect the behaviour of the system. Furthermore multiplying the system matrix from the left by

$$
\left[\begin{array}{cc}
M & 0 \\
0 & I
\end{array}\right]
$$

can be considered as a "reformulation of constraints" in the terminology of Schumacher (1988) and this proves that the resulting representation is externally equivalent to the original one. In the same way, right multiplication by

$$
\left[\begin{array}{ll}
N & Y \\
0 & I
\end{array}\right]
$$

where $E Y=0$ can be considered as a "change of internal variables" [see again Schumacher (1988)]. Finally, left multiplication by

$$
\left[\begin{array}{ll}
I & 0 \\
X & I
\end{array}\right]
$$

where $X E=0$ is a trivial operation: multiplying the equation

$$
\sigma E \xi_{1}=A \xi_{1}+B \xi_{2}
$$

from the left by $X$ gives:

$$
0=X A \xi_{1}+X B \xi_{2} .
$$

This can of course, without affecting the system, be added to the equation

$$
y=C \xi_{1}+D \xi_{2}
$$

yielding

$$
y=(C+X A) \xi_{1}+(D+X B) \xi_{2} .
$$

From the above we can conclude that $(E, A, B$, $C, D)$ and $(\tilde{E}, \tilde{A}, \tilde{B}, \tilde{C}, \tilde{D})$ are externally equivalent.

Before presenting the main theorem of this section, we need the following lemma.

Lemma 6. Let a minimal $\mathbf{D}$ representation be given by $(E, A, B, C, D)$. Decompose the equation space $X$ as $X_{1} \oplus X_{2}$ where $X_{1}=\operatorname{im} E$. Decompose the input space $U$ as $\pi_{U} W^{0} \oplus U_{2}$. Accordingly write

$$
B=\left[\begin{array}{ll}
B_{11} & B_{12} \\
B_{21} & B_{22}
\end{array}\right]
$$

Then $B_{22}$ is invertible.

Proof. Since $(E, A, B, C, D)$ is minimal, Theorem 3 yields that

$$
\operatorname{codim} \operatorname{im} E=\operatorname{codim} \pi_{U} W^{0} .
$$

From this it follows immediately that $B_{22}$ is square. Next we prove that $B_{22}$ is injective. Let $u \in U_{2}$ be such that $B_{22} u=0$. Then $B u \in \operatorname{im} E$, so $u \in B^{-1}[\operatorname{im} E]$. Using the equality

$$
B^{-1}[\operatorname{im} E]=\pi_{U} W^{0}
$$

(Lemma 3) it follows that $u=0$ from which it can be concluded that $B_{22}$ is injective. This proves that $B_{22}$ is invertible.

Theorem 5. Let $(E, A, B, C, D)$ and $(\tilde{E}, \tilde{A}, \tilde{B}$, $\tilde{C}, \bar{D})$ be $\mathbf{D}$ representations that are minimal under external equivalence. Then the two representations are externally equivalent if and only if they are related by operations of strong equivalence.

Proof. The "if" part follows immediately from the above proposition. In order to prove the "only if" part, it should be noted that we can arrive at $\mathbf{D}$ representations of the form (2.8) by using operations of strong equivalence. For that reason we can assume that our $\mathbf{D}$ representations $(E, A, B, C, D)$ and $(\tilde{E}, \tilde{A}, \tilde{B}, \tilde{C}, \tilde{D})$ are already of that form. Next apply Algorithm 2 while using the decomposition of $U$ from the above lemma. This yields externally equivalent $\mathbf{P}$ representations $(F, G, H)$ and $(\bar{F}, \tilde{G}, \tilde{H})$ that are minimal by Lemma 5 . We can now use existing knowledge on the transformation group for externally equivalent minimal $\mathbf{P}$ representations. According to Proposition 2, there exist invertible matrices $S$ and $T$ such that

$$
\left[\begin{array}{ll}
S & 0 \\
0 & I
\end{array}\right]\left[\begin{array}{c}
s G-F \\
H
\end{array}\right]=\left[\begin{array}{c}
s \bar{G}-\bar{F} \\
\tilde{H}
\end{array}\right] T .
$$

Writing this in further detail gives:

$$
\begin{aligned}
& {\left[\begin{array}{llll}
S & 0 & 0 & 0 \\
0 & I & 0 & 0 \\
0 & 0 & I & 0 \\
0 & 0 & 0 & I
\end{array}\right]\left[\begin{array}{cccc}
I & -B_{12} B_{22}^{-1} & 0 & 0 \\
0 & D_{2} B_{22}^{-1} & I & 0 \\
0 & 0 & 0 & I \\
0 & B_{22}^{-1} & 0 & 0
\end{array}\right] } \\
& \times\left[\begin{array}{ccc}
s I-A_{11} & -A_{12} & -B_{11} \\
-A_{21} & 0 & -B_{21} \\
C_{1} & C_{2} & D_{1} \\
0 & 0 & I
\end{array}\right] \\
&= {\left[\begin{array}{cccc}
I & -\tilde{B}_{12} \tilde{B}_{22}^{-1} & 0 & 0 \\
0 & \tilde{D}_{2} \tilde{B}_{22}^{-1} & I & 0 \\
0 & 0 & 0 & I \\
0 & \tilde{B}_{22}^{-1} & 0 & 0
\end{array}\right] } \\
& \times\left[\begin{array}{ccc}
s I-\tilde{A}_{11} & -\tilde{A}_{12} & -\tilde{B}_{11} \\
-\tilde{A}_{21} & 0 & -\tilde{B}_{21} \\
\tilde{C}_{1} & \tilde{C}_{2} & \tilde{D}_{1} \\
0 & 0 & I
\end{array}\right]\left[\begin{array}{lll}
T_{1} & T_{2} & T_{3} \\
T_{4} & T_{5} & T_{6} \\
T_{7} & T_{8} & T_{9}
\end{array}\right] .
\end{aligned}
$$


It now follows immediately that

$$
\begin{aligned}
& T_{1}=S, \\
& T_{2}=T_{3}=T_{7}=T_{8}=0, \\
& T_{9}=I,
\end{aligned}
$$

and $T_{5}$ is invertible. It is easily checked that this implies that also

$$
\begin{aligned}
& {\left[\begin{array}{lll}
S & 0 & 0 \\
0 & I & 0 \\
0 & 0 & I
\end{array}\right]\left[\begin{array}{ccc}
I & -B_{12} B_{22}^{-1} & 0 \\
0 & D_{2} B_{22}^{-1} & I \\
0 & B_{22}^{-1} & 0
\end{array}\right] } \\
& \times\left[\begin{array}{cccc}
s I-A_{11} & -A_{12} & -B_{11} & -B_{12} \\
-A_{21} & 0 & -B_{21} & -B_{22} \\
C_{1} & C_{2} & D_{1} & D_{2}
\end{array}\right] \\
&= {\left[\begin{array}{ccc}
I & -\tilde{B}_{12} \tilde{B}_{22}^{-1} & 0 \\
0 & \tilde{D}_{2} \tilde{B}_{22}^{-1} & I \\
0 & \tilde{B}_{22}^{-1} & 0
\end{array}\right] } \\
& \times\left[\begin{array}{cccc}
s I-\tilde{A}_{11} & -\tilde{A}_{12} & -\tilde{B}_{11} & -\tilde{B}_{12} \\
-\tilde{A}_{21} & 0 & -\tilde{B}_{21} & -\tilde{B}_{22} \\
\tilde{C}_{1} & \tilde{C}_{2} & \tilde{D}_{1} & \tilde{D}_{2}
\end{array}\right] \\
& \times\left[\begin{array}{cccc}
S & 0 & 0 & 0 \\
T_{4} & T_{5} & T_{6} & 0 \\
0 & 0 & I & 0 \\
0 & 0 & 0 & I
\end{array}\right] .
\end{aligned}
$$

Multiplying from the left by

$$
\left[\begin{array}{ccc}
I & -\tilde{B}_{12} \tilde{B}_{22}^{-1} & 0 \\
0 & \tilde{D}_{2} \tilde{B}_{22}^{-1} & I \\
0 & \tilde{B}_{22}^{-1} & 0
\end{array}\right]^{-1}=\left[\begin{array}{ccc}
I & 0 & \tilde{B}_{12} \\
0 & 0 & \tilde{B}_{22} \\
0 & I & -\tilde{D}_{2}
\end{array}\right]
$$

yields

$$
\begin{aligned}
& {\left[\begin{array}{ccc}
S & -S B_{12} B_{22}^{-1}+\tilde{B}_{12} B_{22}^{-1} & 0 \\
0 & \tilde{B}_{22} B_{22}^{-1} & 0 \\
0 & D_{2} B_{22}^{-1}-\tilde{D}_{2} B_{22}^{-1} & I
\end{array}\right] } \\
& \times\left[\begin{array}{cccc}
s I-A_{11} & -A_{12} & -B_{11} & -B_{12} \\
-A_{21} & 0 & -B_{21} & -B_{22} \\
C_{1} & C_{2} & D_{1} & D_{2}
\end{array}\right] \\
&= {\left[\begin{array}{cccc}
s I-\tilde{A}_{11} & -\tilde{A}_{12} & -\tilde{B}_{11} & -\tilde{B}_{12} \\
-\tilde{A}_{21} & 0 & -\tilde{B}_{21} & -\tilde{B}_{22} \\
\tilde{C}_{1} & \tilde{C}_{2} & \tilde{D}_{1} & \tilde{D}_{2}
\end{array}\right] } \\
& \times\left[\begin{array}{cccc}
S & 0 & 0 & 0 \\
T_{4} & T_{5} & T_{6} & 0 \\
0 & 0 & I & 0 \\
0 & 0 & 0 & I
\end{array}\right] .
\end{aligned}
$$

\section{Defining}

$$
\begin{aligned}
M & =\left[\begin{array}{cc}
S & -S B_{12} B_{22}^{-1}+\tilde{B}_{12} B_{22}^{-1} \\
0 & \tilde{B}_{22} B_{22}^{-1}
\end{array}\right], \\
N & =\left[\begin{array}{cc}
S & 0 \\
T_{4} & T_{5}
\end{array}\right],
\end{aligned}
$$

$$
X=\left[\begin{array}{ll}
0 & D_{2} B_{22}^{-1}-\tilde{D}_{2} B_{22}^{-1}
\end{array}\right] \text { and } Y=\left[\begin{array}{cc}
0 & 0 \\
T_{6} & 0
\end{array}\right]
$$

we see that $M$ and $N$ are invertible and

$$
\begin{aligned}
& {\left[\begin{array}{cc}
M & 0 \\
X & I
\end{array}\right]\left[\begin{array}{cc}
s E-A & -B \\
C & D
\end{array}\right]} \\
& =\left[\begin{array}{cc}
s \tilde{E}-\tilde{A} & -\tilde{B} \\
\tilde{C} & \tilde{D}
\end{array}\right]\left[\begin{array}{cc}
N & Y \\
0 & I
\end{array}\right] .
\end{aligned}
$$

This proves that the representations are related by operations of strong equivalence.

\section{CONCLUSIONS}

In this paper we have characterized the minimality of a descriptor representation in terms of the matrices $E, A, B, C$ and $D$. The conditions that are necessary and sufficient for minimality under external equivalence can be summarized as absence of nondynamic modes; controllability and observability at infinity; and observability at finite modes.

These conditions coincide with the conditions for minimality under input-output equivalence that were derived by Grimm (1988), except for the fact that controllability at the finite modes is not required in our case. This is to be expected since the main difference between external equivalence and input-output equivalence is the way in which the uncontrollable modes are treated; the uncontrolled behaviour, i.e. the set of time trajectories of the output variables that are not influenced by the input variables, remains invariant under external equivalence whereas it can be removed under input-output equivalence.

Next we have obtained the transformation group for minimal descriptor representations. We found that the transformations under which minimal descriptor representations are externally equivalent coincide with the operations of strong equivalence as introduced by Verghese et al. Combining this result with the results of our previous paper (Kuijper and Schumacher, 1990) we can conclude that the realization procedure of that paper leads to a minimal descriptor representation that is unique up to operations of strong equivalence.

The theorem on the transformation group can be considered as a version of the state space isomorphism theorem for descriptor representations under external equivalence. It should be noted that the operations of strong equivalence are more complicated than the similarity transformations in the standard state space case. For this reason one might prefer to use other representations such as the pencil representation whose transformation group un- 
der external equivalence consists of isomorphisms. The pencil representation was a basic tool in this paper. Results on minimality and transformations for pencil representations were exploited. The importance of the pencil form stems from the fact that it is closely related to the "autoregressive" (matrix fractional) form, as shown in Kuijper and Schumacher (1990).

\section{REFERENCES}

Aplevich, J. D. (1981). Time-domain input-output representations of linear systems. Automatica, 17, 509-522.

Aplevich, J. D. (1985). Minimal representations of implicit linear systems. Automatica, 21, 259-269.

Ferreira, P. M. G. (1987). On system equivalence. IEEE Trans. Aut. Control AC-32, 619-621.

Grimm, J. (1984). Application de la théorie des systèmes implicites a l'inversion des systèmes. In Bensoussan, A. and J. L. Lions (Eds.), Analysis and Optimization of Systems. Lecture Notes in Control and Information Science, 63, Springer, Berlin (Proc. 6th Int. Conf., Nice, 1984; part 2), pp. 142-156.

Grimm, J. (1988). Realization and canonicity for implicit systems. SIAM J. Contr. Optimiz., 26, 1331-1347.
Kuijper, M. and J. M. Schumacher (1990). Realization of autoregressive equations in pencil and descriptor form. SIAM J. Contr. Optimiz., 28, 1162-1189.

Lewis, F. L. (1986). A survey of linear singular systems. Circuits Syst. Signal Process., 5, 3-36.

Luenberger, D. G. and A. Arbel (1978). Singular dynamic Leontief models. Econometrica, 45, 473-481.

Pugh, A. C., G. E. Hayton and P. Fretwell (1987). Transformations of matrix pencils and implications in linear systems theory. Int. J. Control, 45, 529-548.

Rosenbrock, H. H. (1974a). Non-minimal LCR multiports. Int. J. Control, 20, 1-16.

Rosenbrock, H. H. (1974b). Structural properties of linear dynamical systems. Int. J. Control, 20, 191-202.

Schumacher, J. M. (1988). Transformations of linear systems under external equivalence. Lin. Alg. Applic., 102, 1-34.

Verghese, G., B. C. Lévy and T. Kailath (1981). A generalized state space for singular systems. IEEE Trans. Aut. Control, AC-26, 811-831.

Willems, J. C. (1983). Input-output and state space representations of finite-dimensional linear time-invariant systems. Lin. Alg. Applic., 50, 581-608.

Willems, J. C. (1986). From time series to linear system. Part I: Finite-dimensional linear time invariant systems. Automatica, 22, 561-580.

Willems, J. C. (1989). Models for dynamics. Dynamics Reported, 2, 171-269. 